\title{
AUSTRALASIAN JOURNAL OF PARAMEDICINE \\ Research \\ A simulated night shift for undergraduate paramedicine students: Lessons learnt and the perceived value towards learning
}

Alecka Miles MEH, is a paramedic and Lecturer ${ }^{1}$; Sara Hansen MSN, is Simulation Education Coordinator ${ }^{1}$; Brennen Mills PhD, is Lecturer in Paramedicine ${ }^{1}$

Affiliations:

${ }^{1}$ School of Medical and Health Sciences, Edith Cowan University, Perth, Western Australia

https://doi.org/10.33151/ajp.17.732

\section{Abstract}

\section{Background}

Ensuring undergraduate healthcare students are work ready graduates who possess real-world experiences and realistic expectations of their professional environment is essential for Australian universities. This is becoming increasingly more difficult to accomplish with ongoing difficulties on sourcing appropriate clinical placement learning opportunities. We developed and piloted a simulated paramedic night shift investigating if undergraduate paramedic students perceived the experience to be realistic and to what extent they felt it contributed toward their learning.

\section{Methods}

A total of 24 undergraduate paramedic students were recruited to participate in a simulated 13-hour paramedic night shift held on university campus. Student satisfaction and perceived value towards learning was measured using an online survey the day after the simulation.

\section{Results}

23 of 24 participants completed the online survey. Survey data suggested 22 participants felt the simulated night shift to be realistic of real-life paramedic practice, provided a valuable learning experience and should be made a mandatory component of undergraduate paramedicine curricula.

\section{Conclusion}

Given the difficulties associated with providing clinical placement opportunities for students and the perception among health professionals that on entering the workforce many graduates often lack the adaptability required to undertake shift-work and the professional role of a paramedic, educators should consider the merits of providing overnight simulated experiences to undergraduate students to better prepare and acclimatise students to overnight shift work.

Keywords:

paramedicine; undergraduate education; simulation-based education; medical education; high-fidelity simulation

Corresponding Author: Alecka Miles, a.miles@ecu.edu.au 


\section{Introduction}

Australian university undergraduate paramedic programs have the dual responsibility of training paramedic students in the clinical and professional capabilities required for them to practise immediately after graduating, and to adequately prepare students to adapt to a constantly changing, unpredictable and often stressful workplace environment. There is however, criticism surrounding paramedic and other healthcare professions regarding the efficacy of Australian universities in preparing graduates who are 'work ready' (1-3). In order to be competitive in gaining employment, graduate paramedics must demonstrate that their education has successfully equipped them with the communication, knowledge, professionalism and clinical skills to be deemed 'work ready' as a paramedic $(1,4)$.

In 2010, Willis et al undertook a study to identify what Australian state ambulance service paramedics, undergraduate paramedic students and paramedic academics across six Australian states considered to be work ready in professional paramedic practice (5). This study reported there was disparity in graduate attribute expectations between the paramedic practitioners and academics, with paramedic practitioners expecting students to be able to function immediately in an ambulance in both non-urgent and emergency patient presentations on commencing employment (5). However, paramedics suggested that typically graduates lacked essential communication and interpersonal skills, on-road experience and (often) the maturity required to effectively navigate shift work and the professional role of a paramedic (5). Subsequent studies have highlighted the expectations, experiences and foundations of quality clinical placements for undergraduate paramedic students to prepare them for work readiness $(4,6-10)$ with recent literature advocating that interprofessional placements in diverse clinical settings are the key to improving undergraduate allied health student knowledge and both clinical and soft skills $(2,6)$.

Despite all undergraduate paramedic students having clinical placement experiences embedded in their university education, Willis et al suggest paramedic graduates find the transition to professional practice overwhelming and feel underprepared for their new professional roles upon commencing employment with an emergency ambulance service (5).

Although the challenges and stresses aligned with the transition from undergraduate paramedic student to registered paramedic are sparsely described in the literature, multiple studies from other clinical professions such as nursing and medicine have similarly found that graduates report low feelings of preparedness when they transition to professional practice (11-14). These students report that fear of making mistakes, increased responsibility, lack of confidence in their own clinical competence, facing new situations and environments $(14,15)$ communicating with families (16) and time pressures led to them feeling underprepared on entering the workplace and felt it negatively impacted their ability to deliver quality care (17). Given the unpredictable and uncontrolled environment paramedics work in, it is essential that undergraduate paramedic students are exposed to a range of situations to better ensure their preparedness for their dynamic workplace environment.

A traditional paramedic shift pattern consists of two 10-hour day shifts followed by two 12 to 14-hour night shifts. In the university education setting, paramedic clinical classes and external placements often occur during the day or early evening and are usually of a lesser duration than full paramedic shifts. Undergraduate paramedic training over the course of a night shift is an often-neglected part of university education and may contribute to undergraduate paramedic students being unfamiliar and unprepared for the demands of shift work, in particular night shift, on commencing employment with an ambulance service $(1,18)$.

Providing students with an immersive simulated night shift learning environment, with exposure to the dynamic and fatigue-inducing demands of night shift work, in a safe and controlled setting, has the potential to better ensure undergraduate students are work ready at the time of graduation. The aim of the present study was to develop, trial and undertake a preliminary evaluation of an immersive simulated night shift learning experience, designed specifically for undergraduate paramedicine students, making use of live patient actors as well as realistic simulation scenarios, prehospital settings and equipment.

\section{Methods}

\section{Participants}

A convenience sample of 24 volunteer participants was compiled from the 2017 cohort of students enrolled in a three-year, full-time Bachelor of Science (Paramedicine) at Edith Cowan University (ECU) in Western Australia. The mean age of the participants was 22.8 years (standard deviation = 3.6 ); $63 \%$ were male and $37 \%$ were female. Students were eligible to participate in the study if they were second-year students who were enrolled in the unit entitled Clinical Skills for Paramedic Practice 2. In this unit, the necessary clinical skills and theoretical knowledge required to successfully undertake the simulation-based scenarios for this study were consolidated and/or taught.

Student participants were familiar with simulation-based learning environments, as this style of teaching is frequently used for repetitive practice of clinical assessment and treatment in the Clinical Skills for Paramedic Practice 2 unit, as well as other practical units taught throughout the undergraduate degree. 
However, before the study, students had no exposure at university to full simulated paramedic shifts (day or night), limiting simulation-based exposures to single simulated scenarios.

Recruitment took place at lectures and via online postings on the course and unit learning management system (Blackboard) website. Information provided to the students invited them to participate in a single simulation-based paramedic night shift that would involve scenarios which would build on the theory and clinical skills in their Clinical Skills for Paramedic Practice 2 unit. Informed consent was gathered by participants after attending an information session and receiving an information letter detailing the study background and their entitlements as participants. Participants were randomly assigned into pairs, ie. partners that remained together over the course of the shift, to mimic the traditional paramedic workplace. Due to resources and time constraints, only 24 participants were able to take part in the research. Data was collected on two separate nights during semester 2, 2017.

\section{Ethics}

This project was approved by the ECU Human Research Ethics Committee (\#18112).

\section{Simulation design}

Study investigators used an online survey to ask a convenience sample of 10 currently employed paramedics working in metropolitan Perth to estimate how many jobs they would typically attend on a busy night shift and the most common types of cases they attended. The respondents to this survey reported that the average number of 'jobs attended' was 10 and the most common jobs were developed into carefully designed clinical simulation scenarios (Table 1). Study investigators worked with paramedic educators and simulation experts closely to ensure that tasks participants were expected to perform in the simulations were realistic, within the clinical competency parameters of the participants and could be effectively simulated with existing equipment and infrastructure at ECU.

Table 1. Clinical scenarios undertaken by students during the night shift simulation

\begin{tabular}{|c|c|c|}
\hline No. & Scenario & Initial clinical information \\
\hline 1 & $\begin{array}{l}\text { Dr request } \\
\text { (hospital transfer) }\end{array}$ & $\begin{array}{l}\text { Your patient is an elderly adult person who is complaining of severe pain in their hip area after a } \\
\text { fall at home (aged care facility). The patient is on the floor on arrival with an enrolled nurse (EN) in } \\
\text { attendance. }\end{array}$ \\
\hline 2 & $\begin{array}{l}\text { Shortness of breath } \\
\text { (asthma) }\end{array}$ & $\begin{array}{l}\text { Your patient is a } 29 \text {-year-old person who is complaining of severe shortness of breath. They are in } \\
\text { their bedroom sitting upright. The patient has a history of asthma which is usually well controlled by } \\
\text { Ventolin as directed by their physician. }\end{array}$ \\
\hline 3 & $\begin{array}{l}\text { Assault } \\
\text { (penetrating injury) }\end{array}$ & $\begin{array}{l}\text { Your patient is a } 32 \text {-year-old person who has been stabbed in the upper right thigh after an } \\
\text { altercation with a stranger. They are sitting on the pavement. The patient did not hit their head and } \\
\text { had no loss of consciousness. }\end{array}$ \\
\hline 4 & $\begin{array}{l}\text { Chest pain } \\
\text { (cardiac) }\end{array}$ & $\begin{array}{l}\text { Your patient is a 52-year-old security guard who has been under tremendous stress at home due } \\
\text { to a divorce. They have had a few episodes of mild chest pain over the past few weeks but they } \\
\text { attributed it to stress. This evening while walking around the university campus, intense chest pain } \\
\text { came on suddenly and is radiating up into their jaw. }\end{array}$ \\
\hline 5 & Cardiac arrest & $\begin{array}{l}\text { Your patient was walking home from a party with friends when they collapsed suddenly. The } \\
\text { patient's friend tried to get them to respond and called } 000 \text { when they did not. Bystander CPR was } \\
\text { initiated at the direction of the } 000 \text {-call taker and chest compressions are being performed as you } \\
\text { arrive. CPR has been in progress for } 5 \text { minutes. }\end{array}$ \\
\hline 6 & $\begin{array}{l}\text { Mental health } \\
\text { (threatening } \\
\text { suicide) }\end{array}$ & $\begin{array}{l}\text { Your patient has a history of depression and generalised anxiety disorder. They have recently } \\
\text { broken up with their partner of } 4 \text { years ( } 1 \text { week ago). Since then the patient has been very anxious } \\
\text { and depressed. The patient tried calling their former partner and after they did not answer their calls } \\
\text { they are now threatening to jump from a significant height. }\end{array}$ \\
\hline 7 & Fall $<2 \mathrm{~m}$ & $\begin{array}{l}\text { Your patient is an elderly person who has fallen over at home in their bedroom. They fell onto an } \\
\text { outstretched arm, there is obvious deformity in the forearm, swelling and it is extremely painful. } \\
\text { There is an abrasion and bruising in the area but no signs of the bone breaking the skin. }\end{array}$ \\
\hline 8 & $\begin{array}{l}\text { Traumatic injury } \\
\text { (minor) }\end{array}$ & $\begin{array}{l}\text { Your patient was cutting vegetables and has cut their finger. They state it was bleeding significantly, } \\
\text { however on your arrival there are no signs of active bleeding. }\end{array}$ \\
\hline 9 & $\begin{array}{l}\text { Altered conscious } \\
\text { state }\end{array}$ & $\begin{array}{l}\text { Your patient is a } 20 \text {-year-old person who has been at home drinking alcohol with friends for the past } \\
6 \text { hours. The patient has a past history of diabetes. }\end{array}$ \\
\hline 10 & Generally unwell & $\begin{array}{l}\text { Your patient is a 23-year-old person with cold virus symptoms including, runny nose, sore throat, } \\
\text { and tiredness which they have had for the past two days. }\end{array}$ \\
\hline
\end{tabular}


The presentation order of the 10 scenarios was randomised for the six paramedic crews (paired crews) participating on each night and all participants attended the scenarios in Table 1 at some point during the night shift.

To increase realism, the simulation design included the roles of patients and other relevant scene personnel. These roles were played by volunteer actors who were first-year undergraduate paramedic students enrolled in the core unit entitled Clinical Skills for Paramedic Practice 1.

\section{Pre-simulation preparation}

In the week before each of the scheduled night shift simulations, all participants were required to attend a mandatory information session. Volunteer confederate actors and scenario chaperone/facilitators were also required to attend separate training sessions. Study participants and actors were briefed on the logistics of how the night would run and were informed of the following safety measures to ensure they were prepared for the demands of the night shift:

- In the night leading up to data collection you should ensure you get (at a minimum) 8 hours of sleep. If you have not had at least 8 hours of sleep, you should inform study investigators.

- You should also attempt to nap for 2-3 hours during the day prior to data collection.

- It is imperative you organise your own drop-off and pick-up to and from the university campus before and after data collection. You will not be eligible to take part in the study if you drive to or from ECU on the day of your assigned data collection.

Both study participants and actors were provided with information verbally and in writing regarding the risks associated with sleep deprivation and the possible effects on cognition, the importance of maintaining adequate sleep throughout the course of the study and the increased risk of accidental injury.

\section{Actor and scenario facilitator training}

All confederate actors and scenario facilitators attended an independent 3-hour training session in the week before data collection. This session provided actors with training in how to accurately simulate the injuries and medical ailments associated with clinical presentations of their allocated patients (Table 1) and the scenario facilitators were provided with training on how to objectively facilitate the simulations. This training was essential to ensure all confederate actors and scenario facilitators were familiar with the scenario requirements to maintain consistency in information cues provided to participants across all simulated scenarios. Scenario facilitators also attended and passively observed the confederate actor training to better gauge these information cues and align the presentation of clinical information for each scenario.

\section{Procedure - 'the night'}

The simulated night shift began at 18:00 on a Thursday evening and finished at 07:00 on a Friday morning with 12 participants/ six paramedic crews per night. Participants were dispatched to a 'job' (simulated scenario) and accompanied by a scenario facilitator who led them between scenarios, this included car travel on a pre-determined route inside the university campus. The car travel was undertaken using ECU fleet vehicles which simulated the travel time associated with traveling to a scene from the ambulance station, travel to hospital, and travel back to the ambulance station. In the interest of participant safety over the course of the night shift, the Stanford Sleepiness Scale (19) was used before driving to each scene and on the way back to 'hospital' or 'ambulance station' to ensure drivers were alert enough to drive a vehicle. If a participant operating as the 'driver' reported a degree of sleepiness score of five (foggy; losing interest in remaining awake; slowed down) or more on the scale, the scenario facilitator accompanying the crew was required to walk them back to the 'ambulance station', with the remaining scenario simulations to be undertaken by travelling on foot. At no time during the study did this occur.

On entering each simulation scene, participants were met by a patient and at least one bystander (both confederate actors). The scenario facilitators escorting the participants between scenarios provided important clinical information that was unable to be obtained from visual cues at the scene, assessment of the live patient or from the bystander/s (ie. pulse rate, blood pressure and temperature). Participants were required to assess and treat the patient and to either prepare them for transport to hospital or refer them to an alternate care pathway. Participants were instructed to perform all assessments and non-invasive treatments as they would if they were working to provide professional paramedic services, while invasive treatments (ie. cardiopulmonary resuscitation, cannulation) were undertaken on simulated skills task trainers. Simulations ended once the patient was moved onto the stretcher and ready to be loaded into the 'ambulance'. This triggered facilitators to escort participants back to the car to 'drive to hospital' while the patient would be assisted off the stretcher and would then walk (with the stretcher) to the simulated hospital emergency department where they would then resume waiting in the ambulance triage area on the stretcher in preparation for paramedic handover to the triage nurse. Participants returned to the their 'ambulance station' every 2 hours to restock consumables in their ambulance response bags and attend to personal physical needs before being dispatched to their next job.

\section{Analysis}

The day following data collection, participants were sent a link to an online questionnaire via their student email address. All participants who completed the survey did so within the hours of 15:00 to 18:00 the day after the night shift simulation. 
Each participant was asked to rate their satisfaction with the simulated night shift, perceived learning value, perceived realism of the night shift, whether (hypothetically) they would be interested in undertaking another simulated night shift that same night, and if the exercise should be incorporated into the standard undergraduate curricula. Questions were asked utilising 5-point Likert scales (1 = strongly disagree; 5 = strongly agree). Respondents also had the opportunity to provide additional comments regarding their experience via an open-ended text box. Likert scale ratings were analysed using descriptive statistics (Microsoft Excel) to summarise perceptions of the simulated night shift. Thematic analysis was used to analyse qualitative statements to open ended questions. Study investigators individually examined the text box responses, reviewed the comments collectively and then agreed on common themes related to the aims of the study.

\section{Results}

A total of $23(96 \%)$ of the participants completed the online survey the day following their simulated night shift. Of these respondents, $100 \%$ indicated they either agreed or strongly agreed with the statement: 'I enjoyed participating in the paramedic night shift simulation'. However, only $61 \%$ either agreed or strongly agreed with the statement: 'Given the opportunity, I would be excited about the concept of completing another simulated paramedic night shift tonight'. The remaining $39 \%$ who did not agree they would be excited to participate again the following night, provided comments via open-ended text box such as:

- 'I am still exhausted from last night'

- ' 'I am still tired and my clinical care would suffer'

- 'Already feeling lethargic. I think my performance would exponentially decrease'.

A high portion (87\%) of respondents agreed or strongly agreed with the statement: 'Did the exercise do a good job of replicating a realistic paramedic night shift?' The remaining $13 \%$ were neutral with no respondents disagreeing with the statement. Regardless of how strongly the participants perceived the realism of the simulation-based night shift, the common themes provided in the text box regarding the most realistic aspects among respondents included the simulation design (specifically the case mix), utilisation of 'live' patient actors and bystanders, driving (to scene, to hospital and back to station), the handover at ED, and the long hours of the simulation. They wrote:

- 'The timings of being at a patient, driving to hospital, giving a handover, driving back to depot'

- 'The actors were great, and it's good not knowing the patients'

- 'There was a large variation of scenarios which allowed us to grasp all types of call outs a paramedic could receive on a night shift. Also, our last scenario was a resus at 6am in the morning. It was quite challenging to complete especially after a full night'.
The theme that emerged in the written responses regarding the unrealistic aspects of the simulated night shift included the simulation taking place in familiar university environment, being unable to assess and treat the patients while in the ambulance on the way to hospital and the absence of a requirement to complete patient care records (PCR):

- '...extending the patient care into the ambulance and on the way to hospital...'

- 'I think having even some PCR paperwork would have been good... and helped with my handovers'.

Nearly all (96\%) of respondents agreed or strongly agreed with the statement: 'I would encourage the university to incorporate this exercise into the standard undergraduate curricula'. The remaining $4 \%$ were neutral with no respondents disagreeing with the statement. When asked the statement: 'Did the exercise provide a valuable learning experience?', $100 \%$ of the respondents agreed or strongly agreed.

The final survey question asked: 'Are there any other comments you would like to make?' Some respondents wrote:

- '...Have a real paramedic there to debrief and correct any thing that was wrong through the night'

- 'The night shift was a valuable learning tool that has helped me to understand what a paramedic night shift is like and also allowed me to push myself and strengthen my skills'.

\section{Discussion}

The present study sought to gauge participant satisfaction, the perceived learning value and the perceived realism of a simulated paramedic night shift among undergraduate paramedicine students, as well as whether students felt the experience was worthy of integration into standard undergraduate curricula. Furthermore, this pilot study aimed to assess the feasibility of providing a 13-hour paramedic night shift with adequate levels of fidelity to be of perceived benefit to students given current equipment and infrastructure available.

With respect to our first study aim, every respondent agreed that the simulated night shift was enjoyable and provided a valuable learning experience. The apparent value to participant learning was further emphasised by their positive responses encouraging incorporation of the experience into the standard undergraduate curricula. Conversely, despite the reported levels of satisfaction and perceived value to learning, few students were enthusiastic about the prospect of completing a second night shift the following night. However, open-ended comments suggested this was mostly attributable to tiredness from the first night, as opposed to a lack of interest from a lack of fidelity or perceived learning value. Interestingly, the typical paramedic roster sees paramedics undertake two back-to-back night or evening shifts of at least 12 hours duration, suggesting that these students would have felt less than optimistic about their willingness and capacity to undertake their next shift. 
This reported lack of motivation to undertake a subsequent night shift highlights that despite a night-shift preparation session and information, the participants need further education strategies to assist them with preparing to effectively navigate shift work and the associated cumulative effects of night shift work on the body's circadian rhythm (21). Although, we do acknowledge a possible alternate interpretation of this data could be that the undertaking of the simulated night shift was a novelty for students which had worn off following its completion.

\section{Of further alarm was some of the participants suggesting} concern over the quality of their clinical care given their fatigue levels if they were required to undertake a second night shift. These comments demonstrate some insight into the perception of their own clinical capabilities, suggesting that potentially putting students outside of their comfort zone by attending two back-to-back simulated night shifts could enhance their understanding of the inherent requirements of shift work. This perhaps reiterates the importance of exposing students to night shift work before their entry into the paramedic workforce so they better understand their own limitations when operating under fatigue. Previous research suggests that inadequate sleep in medical professionals who undertake shift work leads to increases in perceived medical errors and a greater risk of developing stress and depressive conditions early in their careers (21-24).

\section{Strengths and limitations}

This simulation exercise allowed participants to not only experience the lifecycle of each job (scenario) from dispatch to handover at hospital, but also repeating this over the course of a 13-hour long shift as would happen in real-world practice. Furthermore, the simulated nature of the scenarios allowed for consistency across scenarios ensuring all participants received the same learning experience, which is not possible from learning occurring within the clinical placement setting that is reliant on random patient presentation (25-26). Furthermore, the learning experience was able to be provided to undergraduate paramedic students with pre-existing equipment and infrastructure, utilisation of voluntary patient actors (in-kind contribution), and with only a small budget of approximately AUD700 for vehicle fuel.

Study investigators recognise that the data to support this exercise is limited to participant perceptions and that as students volunteered for this study it may be subject to selfselection bias with more motivated students self-selecting into voluntary participation. The participants' university training does not involve them undertaking regular night shifts as part of the degree and their previous experience with night shift is outside of this training is unknown. As such, study investigators acknowledge that the students' reference point for commenting on the realism of the night shift simulation may be limited to perceptions rather than lived experience. Although the research design is innovative, the small sample size due to time and resource constraints restricts our ability to generalise the results to a wider population of undergraduate paramedicine students. Further, the 10 scenarios incorporated in the study design were determined by surveying a small sample of paramedics working in metropolitan Perth and may be greater than the average number of jobs attended in other regions.

These limitations notwithstanding, it is clear that students found value in the experience and felt it would be of benefit for other undergraduate paramedicine students to complete a similar experience to better understand the challenges of overnight paramedic shift work. Future research could seek to re-examine similar simulated experiences, including successive night shifts, with greater sample sizes, ideally with mandatory participation so as to eliminate self-selection biases. It would also be of interest to gauge previous night shift experience and to what extent the fatigue some participants suggested they experienced over the course of the simulated night shift impacted on their clinical performance, given some of the open-ended comments provided in our feedback survey. A combination of videoing each simulation and using student participant self-assessment, peer-assessment and selfreflection of clinical performance in the weeks following the simulations, may also work to further enhance student learning. This is worth considering as an inclusion and improvement to future study designs in this space.

\section{Conclusion}

Embedding realistic night shift training through simulation not only replicates real life practice, but also has the ability to provide a high level of consistent clinical practice exposure across the course of an undergraduate degree, something that has previously been identified as an uncontrollable deficit of the traditional clinical placement model $(25,26)$. This research advocates for the inclusion of simulated night shifts into the standard undergraduate curricula as it is perceived by students to be realistic, enjoyable and to provide a valuable learning experience. Further, while such training does require an investment of in-kind resources, it can likely be provided by most education institutions with minimal extraneous costs. With changes to higher education funding as a result of federal budget cuts to the sector, vigilant consideration needs to go into undergraduate course and unit design, and budget allocations need to consider where to best allocate funds to improve the education and training of our undergraduate students (27). Ideally, these outcomes should be repeated via a larger-scale, preferably multi-site, replication study before educators invest heavily in implementing changes into curricula at the expense of other teaching and learning activities.

\section{Acknowledgements}

The authors thank ECU for their support of this project and the assistance and hard work of Travis Cruickshank, Danielle Bartlett, Tim Rankin, Will Errey, Matthew Gaston, Taylor Hay, Dirk Du Ploy, Zahed Minhaj, Tyron Rumble, Pauline Zaenker, Jono Goh and Gavin Mazzuchelli. 


\section{Competing interests}

The authors have no conflicts of interest to declare. Each author of this paper has completed the ICMJE conflict of interest statement.

\section{References}

1. O'Brien K, Moore A, Hartley P, et al. Lessons about work readiness from final year paramedic students in an Australian university. Australasian Journal of Paramedicine 2014;10(4). Available at: https://ajp.paramedics.org/index. php/ajp/article/view/52/44

2. Thompson J, Grantham H, Houston D. Paramedic capstone education model: Building work ready graduates. Australasian Journal of Paramedicine 2015;12(3). Available at: https://ajp.paramedics.org/index.php/ajp/article/ view/15/487

3. Gibson B, Brightwell R. The developments in paramedical science and the implications of national and international accreditation in alliance with ambulance authorities. Paper presented at the EDU-COM 2006 Engagement and Empowerment, Thailand.

4. Waxman A, Williams B. Paramedic pre-employment education and the concerns of our future: What are our expectations? Australasian Journal of Paramedicine 2006;4(4).

5. Willis $E$, Williams B, Brightwell RF, et al. Road-ready paramedics and the supporting sciences curriculum. FoHPE 2010; 11(2):1-13. Available at: https://researchoutput.csu. edu.au/ws/portalfiles/portal/8760855/14860postpub.pdf

6. Hou XY, Rego J, Service M. Paramedic education opportunities and challenges in Australia. Emerg Med Australas 2013;25:114-19. Available at: https://onlinelibrary. wiley.com/doi/full/10.1111/1742-6723.12034

7. McCall L, Wray N, Lord B. Factors affecting the education of pre-employment paramedic students during the clinical practicum. Australasian Journal of Paramedicine 2014;7(4). Available at: https://ajp.paramedics.org/index.php/ajp/article/ view/189/200

8. Williams B, Brown T, Winship C. The mismatch between perceived and preferred expectations of undergraduate paramedic students. J Allied Health 2012;10(4). Available at: https://nsuworks.nova.edu/ijahsp/vol10/iss4/12/

9. Health and Care Professional Council. Standards of education and training guidance. London, UK: Health and Care Professionals, 2012. Available at: www.hcpc-uk.org/ globalassets/resources/guidance/standards-of-educationand-training-guidance.pdf

10.O'Meara P, Hickson H, Huggins C. Starting the conversation: What are the issues for paramedic student clinical education? Australasian Journal of Paramedicine 2014;11(4). Available at: https://ajp.paramedics.org/index. php/ajp/article/view/4/108
11. Stroben F, Schroder T, Dannenberg KA, et al. A simulated night shift in the emergency room increases students' selfefficacy independent of role taking over during simulation. BMC Med Educ 2016; 16:177. Available at: https:// bmcmededuc.biomedcentral.com/articles/10.1186/s12909016-0699-9

12.Goldacre MJ, Taylor K, Lambert TW. Views of junior doctors about whether their medical school prepared them well for work: questionnaire surveys. BMC Med Educ 2010;10:8. Available at: https://bmcmededuc.biomedcentral.com/ articles/10.1186/1472-6920-10-78

13.Cave J, Woolf $\mathrm{K}$, Jones $\mathrm{A}$, et al. Easing the transition from student to doctor: how can medical schools help prepare their graduates for starting work? Med Teach 2009;31:403-8. Available at: www.tandfonline.com/doi/ full/10.1080/01421590802348127

14. Oermann MH, Garvin MF. Stresses and challenges for new graduates in hospitals. Nurs Educ Today 2002;22:225-30. Available at: www.sciencedirect.com/science/article/pii/ S0260691701906951?via\%3Dihub

15.Delaney C. Walking a fine line: graduate nurses' transition experiences during orientation. J Nurse Educ 2003;42:43743.

16.Duchescher JEB. Out in the real world: newly graduated nurses in acute-care speak out. J Nurse Adm 2001;31:42639.

17. Maben J, Latter S, Clark JM. The theory-practice gap: impact of professional-bureaucratic work conflict on newlyqualified nurses. J Adv Nurs 2006;55:465-77. Available at: https://onlinelibrary.wiley.com/doi/full/10.1111/j.13652648.2006.03939.x

18. Kaihlanen A-M, Strandell C, Salminen L. Towards unpredictable - the anticipations of nursing students of the transition to a registered nurse. Nurs Palliat Care 2016;1:946. Available at: www.oatext.com/Towards-unpredictable-The-anticipations-of-nursing-students-of-the-transition-to-aregistered-nurse.php

19. Maclean A, Fekken G, Saskin P, et al. Psychometric evaluation of the Standford Sleepiness Scale. J Sleep Res 1992;1:35-9. Available at: https://onlinelibrary. wiley.com/doi/abs/10.1111/j.1365-2869.1992. tb00006.x?sid=nlm\%3Apubmed

20.Borbély AA, Daan S, Wirz】Justice A, Deboer T. The two[ process model of sleep regulation: a reappraisal. J Sleep Res 2016;25:131-43.

21. Kalmbach DA, Arnedt T, Song PX, et al. Sleep disturbances and short sleep risk factors for depression and perceived medical errors in first-year residents. Sleep 2017;40. Available at: www.ncbi.nlm.nih.gov/pmc/articles/ PMC6084763/

22. Kalmbach DA, Pillai V, Cheng P, et al. Shift work disorder, depression and anxiety in the transition to rotating shifts: the role of sleep reactivity. Sleep Med 2015;16:1532-8. Available at: www.ncbi.nlm.nih.gov/pmc/articles/ PMC4803034/ 


\section{References (continued)}

23. Saadat $H$, Sissonnette $B$, Tumin $D$, et al. Time to talk about work-hour impact on anaesthesiologists: the effects of sleep deprivation on profile of mood sates and cognitive tasks. Paediatr Anaesth 2015;26:66-71. Available at: https:// onlinelibrary.wiley.com/doi/full/10.1111/pan.12809

24. Ramey S, MacQuarrie A, Cochrane A, et al. Drowsy and dangerous? Fatigue in paramedics: an overview. Irish Journal Paramedicine 2019;4(1). Available at: www. irishparamedicine.com/index.php/ijp/article/view/175 25.Mills BW, Carter OBJ, Rudd CJ, et al. Quantification of opportunities for early-stage paramedicine students to practice clinical skills during clinical placements compared to an equal dose of simulation-based workshops. BMJ Simul Technol Enhanc Learn 2015;1:24-8.

26. Boyle MJ, William B, Cooper J, et al. Ambulance clinical placements - a pilot study of students' experience. BMC Med Educ 2008;8:19.

27.Probert $B$. The quality of australia's higher education system: how it might be defined, improved and assured. Sydney, New South Wales, Australia: Australian Government Office for Learning and Teaching, 2015. Available at: https://nla.gov.au/nla.obj-310528240/view 